\title{
A Novel Lane Line Detection Based on Multi-feature Fusion and Windows Searching
}

\section{Chuangxin Cai}

Huaiyin Institute of Technology

Shangbing Gao ( $\square$ luxiaofen_2002@126.com )

Huaiyin Institute of Technology

\section{Zhigeng Pan}

Hangzhou Normal University

Hao Zheng

Nanjing Xiaozhuang University

Zihe Huang

Huaiyin Institute of Technology

\section{Research Article}

Keywords: Intelligent vehicle, automatic driving, lane detection, multi-feature fusion, homography transformation

Posted Date: April 28th, 2021

DOl: https://doi.org/10.21203/rs.3.rs-428581/v1

License: (c) (i) This work is licensed under a Creative Commons Attribution 4.0 International License.

Read Full License 


\title{
A Novel Lane Line Detection Based on Multi- feature Fusion and Windows Searching
}

\author{
Chuangxin Cai, ${ }^{1}$ \\ Shangbing Gao, ${ }^{1 *}$ \\ Email luxiaofen_2002@126.com \\ Zhigeng Pan, ${ }^{2}$ \\ Hao Zheng, ${ }^{3 *}$ \\ Email 30741989@qq.com
}

Zihe Huang, 1

1 Faculty of Computer and Software Engineering, Huaiyin Institute of Technology, Huaian 223003

2 P.R.China Institute of VR and Intelligent System, Hangzhou Normal University, Hangzhou 311121

3 P.R.China Key Laboratory of Intelligent Information Processing, Nanjing Xiaozhuang University, Nanjing, 211171, P.R. China

\begin{abstract}
Lane detection embedded in intelligent vehicles can greatly improve the security of automatic driving. This work offers a new approach towards lane detection in the video in real-time combining multi-feature fusion and window searching. As the pre-procession, polygon filling is adopted to locate ROI (Region of Interest) in the video frames, which contain the lane-lines to be detected. To remove the backgrounds in the ROIs, we extract and fuse the features of color, histogram, and gradient of line lanes. Based on the density distribution of the pixels in the line lanes, the initial location is found by homography transformation. Then all candidate pixel points in the whole lane-line are extracted in the way of window searching. Finally, On the basis of the obtained lane-mark coordinates, a curve model is defined, and the model parameters are obtained by Least Square Estimation (LSE). Experimental results show the robustness and instantaneity of the proposed algorithm with the accuracy of $96 \%$ and the detecting time of only $20.7 \mathrm{~ms}$. In addition, lane-lines with misleading backdrops can also be detected such as yellow lane lines on the ground, shadow, bright light, lane-line defects and traffic light
\end{abstract}

Keywords: Intelligent vehicle; automatic driving; lane detection; multi-feature fusion; homography transformation

\section{Introduction}

The unceasing progress in China's economy and urbanization have enhanced internal expressway mileage and automobiles, and resulted in great traffic jams. Besides, with the requirements of comfortable driving, Advanced Driver Assistance Systems (ADAS) and autonomous driving techniques have attracted widespread attention from domestic and foreign scholars. In recent years, the major companies such as Baidu, General Motors, Google and Uber have delved into Autonomous Vehicles (AVs), with higher mobility, fewer faults and smarter choices. As a key of autonomous driving technology, lane detection analyses the position of the vehicle on the road by extracting lane information from the image. It provides a decision basis for the steering and lane change of the vehicle.

At present, there are two main methods for lane detection: Deep Learning (DL) [1-3] and traditional feature extraction. DL mainly uses a multi-layer convolutional neural network and a large number of samples are used to complete lane line recognition through continuous learning, without the need of manually designed features. The method has the advantages of strong robustness and high accuracy, but the biggest defect of high computational complexity and large time overhead, which requires GPU for supporting. In contrast, traditional methods focus on the characteristics of the lane line itself, such as the color, texture, brightness and the type of lane line to separate 
lane lines from the complex background [4-5]. For example, Nima et al. [6] covert RGB color space into YCbCr space and HSV space, and implement lane detection by the gradient detection operator. Jamel Baili et al. [7] use a horizontal difference filter to simplify the edge detection process and group the detected edge points into a straight line by the improved Hough transformation. Qian Kidd et al. [8] utilize the Sobel edge detection operator to detect lane edge information, and detect possible lane line by the modified Hough transform on the region of interest. However, the above three lane detection methods are susceptible to illumination, road shadow, and lane line defects. Under such defective conditions, the lane curves are hardly detected and the time overhead is large.

In this paper, we propose a novel lane detection method by combining multi-feature fusion and window searching. The main contribution of the proposed method is summarized as follows: 1) the color, gradient and grayscale features of lane line are considered. Then, the multi-feature fusion algorithm is proposed to fuse these features, creating a binary image and enhancing the robustness of lane detection in complex environment. compared with the deep learning features. Compared with deep features, the time complexity of extracting these three features is greatly reduced, and the accuracy is higher; 2) If the left and right lane are detected in the previous frame of the video image, The LFPF (Line Fits by Previous Fits) method is proposed to search lane-line pixels. It uses the relationship between the front and back frames, and the lane-line pixels in the current frame are searched in the vicinity of the left and right lane-line boundary equations fitted in the previous frame, which can effectively reduce the time complexity of the algorithm and improve stability of lane detection.

\section{Proposed Method}

The algorithm mainly includes Image Pre-procession, Multi-Feature Fusion, Lane-line Fitting and Lane-line area visualization. The overall architecture of the proposed method is shown in Fig. 1.

In Image Pre-procession, the video frames are resized firstly. Different angles of the chessboard images are used for camera distortion coefficients. The correspondence between 3D word and 2D image points? for a bunch of images is stored and applied to the actual camera. Finally, the polygon-filling is used to obtain a trapezoidal ROI (Region of interest), which contains lane-lines to be detected so as to avoid the unnecessary calculation.

In the module of Multi-Feature Fusion, color space conversion, histogram equalization, and gradient operator filtering are performed on the pre-processed images. Then the logical OR operation is used on the pixels of the image. At last, a binary image is obtained by morphological filtering and threshold process. After the above steps, the lane-line is separated from the complex background.

In Lane-line Fitting, homography transformation is performed on the image to obtain a planar lane-line binary image. Then, some slide windows are used to search lane line pixels. Finally, the quadratic polynomial fitting is performed on the pixel coordinates to determine the left and right lane boundary equations.

Lane-line area visualization: the pixels within the boundary of the left and right lane lines are color-marked, and then the color-marked image is projected to the original image through perspective transformation to complete the visualization of lane line detection and tracking.

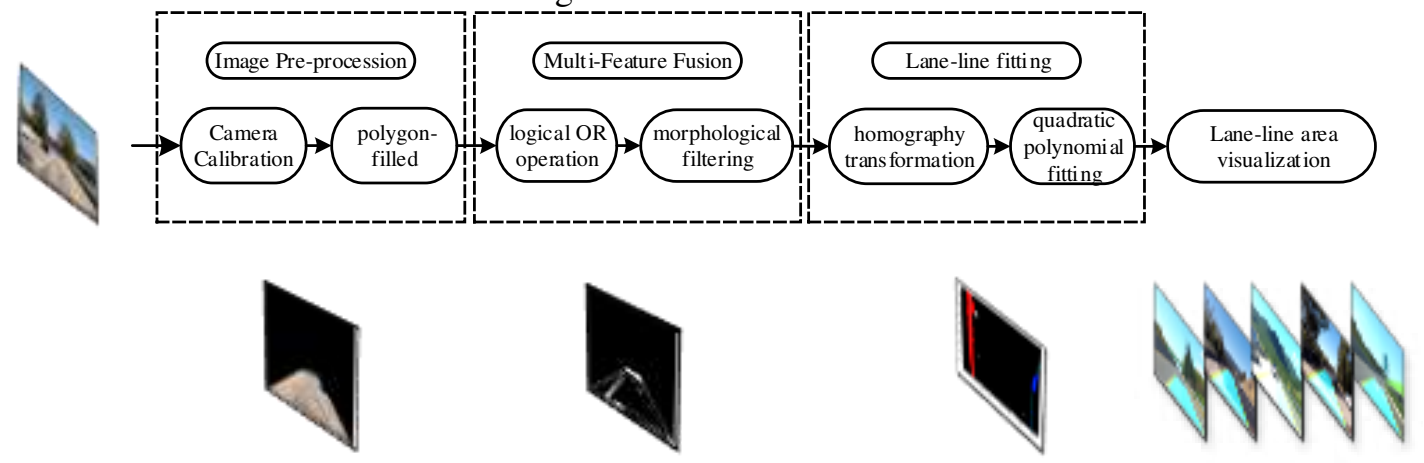

Figure 1 Flowchart of proposed lane detection method.

\section{Multi-feature Fusion}

The white lane-line and the road surface gray value are too close to extracting the lane information after the grayscale processing when the image captured by the camera is too bright or too dark. Furthermore, the street tree will generate a shadow under the illumination of the sun, so that the information of the lane-line is lost when it is covered on the white lane. Finally, the significance of the yellow lane is reduced and cannot be separated from the road surface background after the image is grayed. In order to address the above problems, the multi-feature fusion algorithm is used to process the image.

\subsection{Color Transforms}


When the light is relatively stronger, the yellow lane lines in the RGB color space appear less obvious than the road surface after graying. Therefore, A. Ajmal [9] applied H-V or S-V channels to improve the quality of object saliency detection. According to [9], the color space of the preprocessed image is converted from RGB to HSV in this paper. The HSV model focuses on color representation. By setting a certain range of hue $(\mathrm{H})$, saturation $(\mathrm{S})$, and lightness (V). HSV model performs better on the segmentation of specified colors. The setting formula is as follows:

$$
O(x, y)= \begin{cases}1, & H_{\min }<h(x, y)<H_{\max } \text { and } \\ & S_{\min }<s(x, y)<S_{\max } \text { and } \\ & V_{\min }<v(x, y)<V_{\max } \\ 0, & \text { otherwise }\end{cases}
$$

Where $H_{\text {min }}, H_{\max }$ represents the range of Hue values, $S_{\min }, S_{\max }, V_{\min }, V_{\max }$ represent the ranges of Saturation and Value. Fig. 2 shows the extracted yellow lane line. Fig. 2(a) is the pre-processed image, and Fig. 2(b) is the result after threshold procession in HSV color space. It can be seen that the algorithm can effectively detect the yellow lane lines, but the white lane lines are completely ignored.

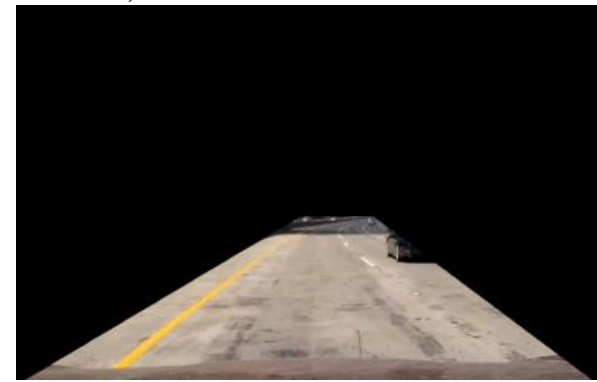

(a)

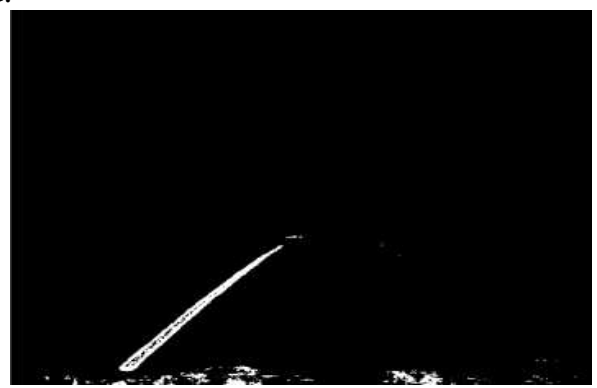

(b)

Figure 2 Yellow lane extraction ((a) Input image with region of interest, (b) Yellow mask).

\subsection{Histogram Equalization}

When the image is dark overall, the white lane in the image is not significant with respect to the road surface after the image is grayed out. Furthermore, the brightness of lane-line is also darkened when the edges are worn. For better segmentation, the image contrast should be enhanced. Generally, methods for improving image contrast include direct contrast enhancement [10] and indirect contrast enhancement [11]. In this paper, histogram equalization, an indirect method is used to enhance the contrast of the image to detect white lane-line. Considering that the lane lines are mostly white, the brightness of the lane lines concentrates is within a certain range. The following formula is used to find a binary image of the white lane:

$$
h(x, y)= \begin{cases}1, & B_{\min }<b(x, y)<B_{\max } \\ 0, & \text { otherwise }\end{cases}
$$

In the formula, $B_{\min }$ and $B_{\max }$ respectively represent the minimum and maximum gray values after the histogram equalization. By testing on thousands of frames under different lighting conditions, $B_{\min }$ and $B_{\max }$ are set to 200 and 250 empirically. Through histogram equalization, the white lane line is effectively detected.

\subsection{Gradient Filtering}

In order to better detect the boundary of the lane-line and facilitate subsequent procession, the Sobel operator is used to filter the image to get the edge information of the lane. Different from the traditional Sobel edge detection algorithm, the image is converted to grayscale. The horizontal and vertical Sobel operators is used to filter the image separately to obtain the images S_h and S_v.

$$
\begin{gathered}
S_{h}=\left[\begin{array}{ccc}
-1 & 0 & 1 \\
-2 & 0 & 2 \\
-1 & 0 & 1
\end{array}\right] \\
S_{v}=\left[\begin{array}{ccc}
1 & 2 & 1 \\
0 & 0 & 0 \\
-1 & -2 & -1
\end{array}\right] \\
S_{h v}=\sqrt{\left(S_{h}\right)^{2}+\left(S_{v}\right)^{2}} / \max \left(\sqrt{\left(S_{h}\right)^{2}+\left(S_{v}\right)^{2}}\right) \times 255
\end{gathered}
$$

In the above functions, $S_{h}$ is horizontal Sobel operator and $S_{v}$ is vertical Sobel operator. Empirically, $S_{h v}$ in (5) is added to obtain the expected edge detection, in which $S_{-}$h and S_v are the images filtered by $S_{h}$ and $S_{v}$. max () is the maximum pixel value. Fig. 3(a) is the pre-processed image, Fig. 3(b) is the filtered image by Sobel operator. It can be seen that the lane line boundaries in the ROI have been precisely detected. 


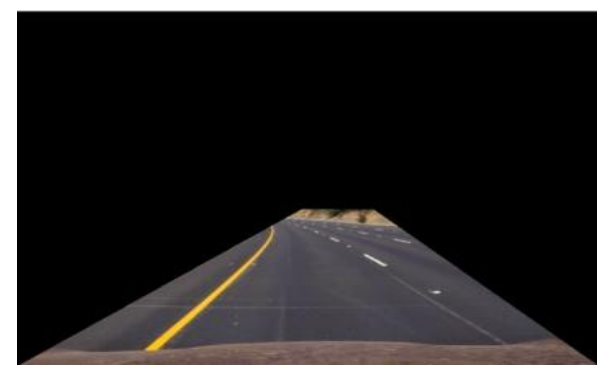

(a)

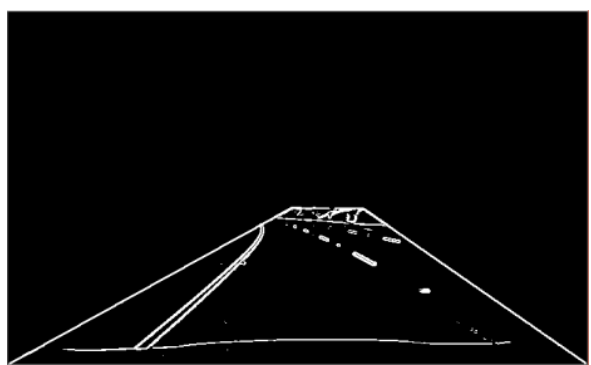

(b)

Figure 3 Image filtered with gradient operator ((a) Input image with region of interest, (b) Sobel operator).

\subsection{Image Fusion}

Correctly creating the binary image from the input frame is the very first step of the whole lane detection. It plays an important role in creating the binary image according to the designed features for detection. Image Fusion uses a specific algorithm to combine two or more images into a new image. The fusion result can use the correlation of two (or more) images in space-time and the complementarity in information, and make the image obtained after fusion have a more comprehensive and clear description of the lane line.

In order to detect the white lane, the histogram of the input frame is equalized to highlight the actual lane-line before threshold process. For the yellow lane, thresholds on the channels of H, S and V is employed in HSV color space. Furthermore, the input frame is convolved with Sobel kernel to get an estimate of the gradients of the lines. Then, the logical OR operation of pixels is performed to fuse the features of color, histogram and gradient. Finally, the morphological closure is used to fill the gaps in the binary image. The image obtained after pixel-level image fusion has more detailed information, such as the extraction of line-lane edges and textures, which is conducive to further analysis, processing, and understanding of the image, and can also expose potential targets, which is conducive to judgment and identification of potential target pixels Point operation, this method can save as much information in the source image as possible so that the content and details of the merged image are increased. The result of the multi-feature fusion algorithm is shown in Fig. 3. Compared with figures 2(b) and 3(b), both yellow and white lane lines are detected in Fig. 4. It can be seen that the Multi-feature fusion algorithm can effectively eliminate noise interference and has good robustness to yellow and defect lane condition

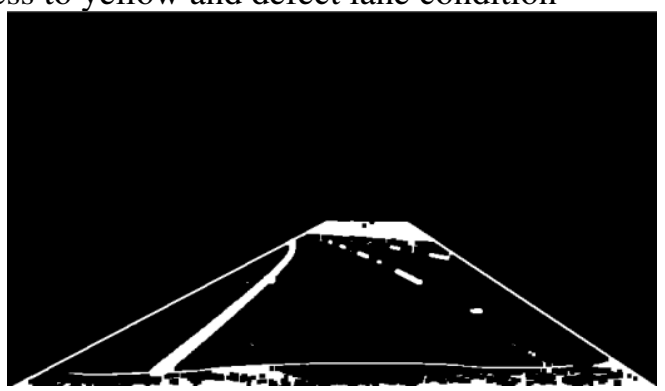

Figure 4 The result of the multi-feature fusion algorithm under road condition.

\section{Line Detection}

At present, straight-line models are mostly used in lane detection algorithms to fit lane-lines. Considering that only color [12-13] or edge [14-15] features are extracted, the model is of low complexity, good real-time performance, and robustness to straight lane-lines. However, this method is limited to the inaccuracy of fitting results, i.e., lanelines can hardly be fitted in the form of curves in practical applications. In this paper, we are seeking a way to fit more precise curves for lane-line detection.

\subsection{Lane Pixel Search}

After the Multi-Feature fusion processing, high-quality binary image of the lane line can be obtained. Then, the wider-range and multi-lane feature data is obtained by converting the binary image into the top-view space via homography transformation. Furthermore, a lane line detection method based on pixel density distribution is proposed in the lane line recognition process, and lane window pixels are extracted using window searching.

In order to identify which pixels of a given binary image belonged to lane-lines, there are two possibilities at least. The first one is never identified where the lane-lines are in a new brand frame, the LFSW (Line Fits by Sliding windows) method is proposed to perform an exhaustive search on the frame. This method starts from the bottom of 
the image, precisely from the peak location of the histogram of the binary image, two windows is slid towards the upper side of the image, decided which pixels belonged to which lane-line. This method has high accuracy, but high time complexity

The second one is confidently identified lane-lines on the previous frame in a video, lane-line pixel search can limit in the neighborhood of the lane-lines detected before, since all the video going at $20 \mathrm{fps}$, the lines won't be so far. The LFPF (Line Fits by Previous Fits) method is proposed to keep track of detected lines across successive frames, which helped faster the lane-line search. The specific steps of the algorithm are as follows:

Step1. The homography transformation is performed on the binary image processed by the multi-feature fusion algorithm since it can map points in one plane to another, which is convenient for subsequent lane-line pixels search with feature extraction windows. Another advantage is lane-lines can be identified even when it is defective. Though this step, the image of homography transformation is obtained, as shown in Fig. 5. White areas are lane line pixels.

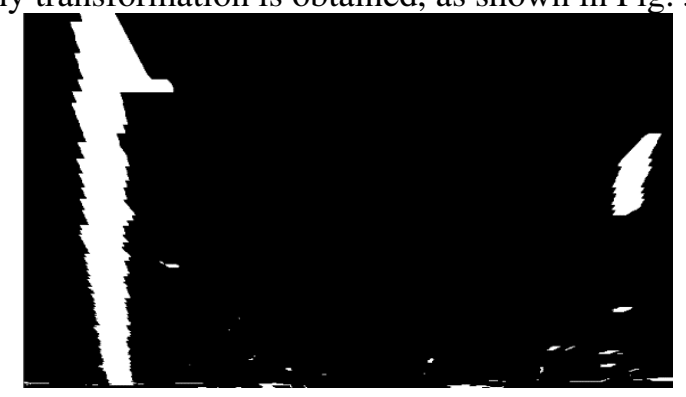

Figure 5 The Image of homography transformation.

Step 2. The density distribution of pixel is counted on the image of homography transformation, as shown in Fig. 6, where the abscissa is the length of Fig. 5 and the ordinate is the density distribution of pixels. Two peaks are formed due to the lane pixels longitudinally distribute and cluster together in a single binary image, and the starting positions SL and SR of the feature extraction window are determined based on the peaks when searching for the left and right lane.

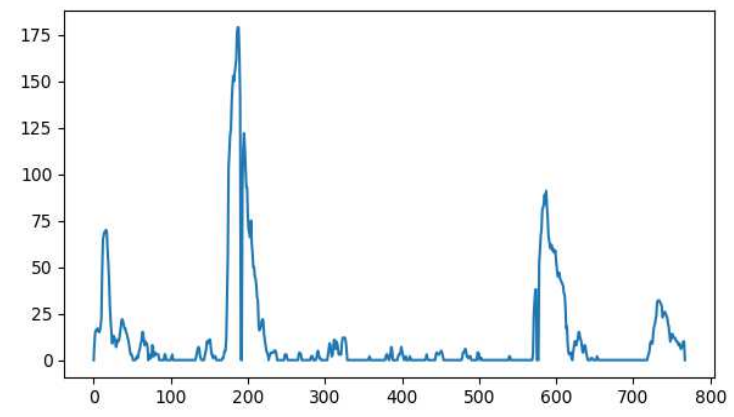

Figure 6 The density distribution of pixel density.

Step 3. After the starting position of the lane-line feature extraction window is obtained, the LFSW method is proposed to search on a frame. In order to avoid the influence of noise around the lane-lines, 12 feature extraction windows are respectively set for the left and right lane, so as to search non-zero pixels in the image vertically from bottom to top. Taking the left lane for example, the starting position of the first window is $S L_{1}$. Secondly, the mean of the abscissa values of all nonzero pixel points in the window is calculated, denoted as $S L_{2}$. Then the starting position of the second window is $S L_{2}$, and so on, until the traversal of 12 windows is completed.

In the practical application of the algorithm, the LFPF is proposed to save time and improve the efficiency of laneline pixels searching, which uses the left and right lane boundary equations fitted in the previous frame to find nonzero pixels. L1 (L2) is used to indicate whether the left (right) lane is detected in the previous frame. Firstly, if the left and right lane were not detected in the previous frame, the feature extraction window is used to find nonzero pixels. In contrast, the left and right lane boundary equations are used to find nonzero pixels, which fitted in the previous frame. The horizontal offset is set to 50. If the left lane boundary equation of the previous frame is $f(x)$, then the search range for new nonzero pixels of the left lane is $[f(x)-50, f(x)+50]$, the search range of the new nonzero pixels of the right lane is similar. The overall algorithm flow of lane-line search is shown in Fig. 7. 


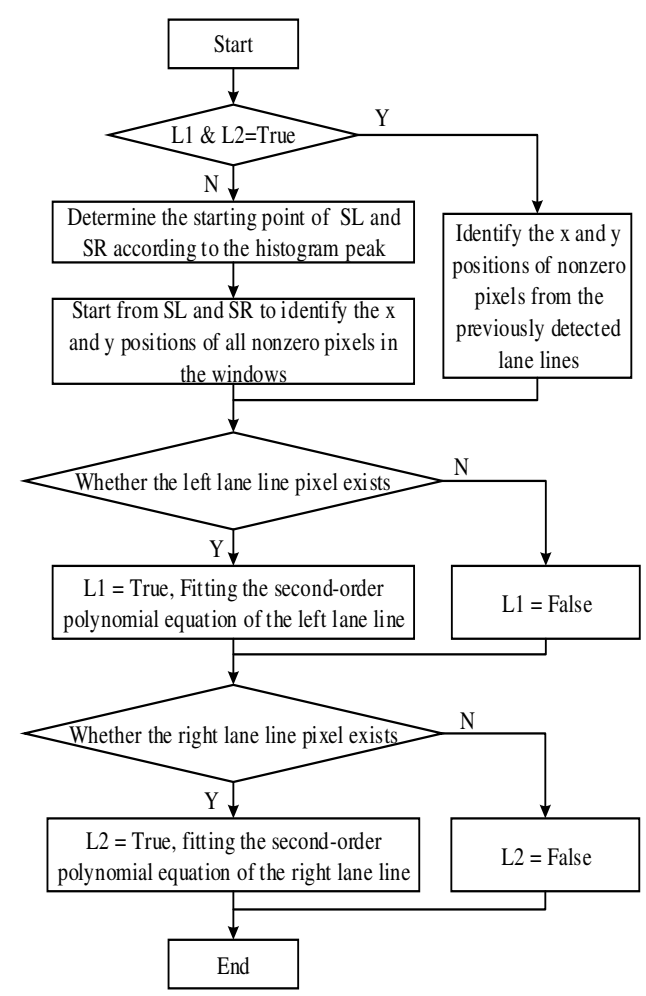

Figure 7 The flow chat of lane-line pixel search algorithm.

\subsection{Lane Line Fitting}

In order to better adapted to the situation where the lane-line is a straight or a curve line, the quadratic polynomial equation is used to improve the robustness of the lane detection, the fitted quadratic polynomial is described as:

$$
x=a+b y+c y^{2}
$$

where $x$ and $y$ is respectively the abscissa and ordinate values of all lane-line pixels obtained by the feature extraction windows, $a, b$ and $c$ are unknown parameters. Assuming that a lane had acquired the coordinates of $\mathrm{N}$ pixels, the set of pixels could be expressed as $\left(x_{i}, y_{i}\right), i=1,2, \ldots, N$. The error function of the least square is identified.

$$
\mathrm{e}=\sum_{i=1}^{N}\left(\left(x_{i}-\left(a+b y_{i}+c y_{i}^{2}\right)\right)^{2}\right.
$$

The least square approach is applied to get the unknowns parameters $a, b$ and $c$ in equation. Finally, the boundary equation of the lane-line can be determined, as shown in Fig. 8.

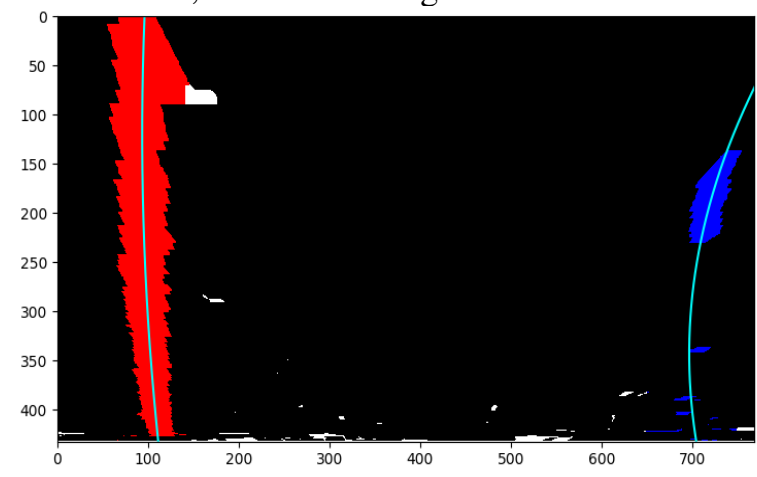

Figure 8 The lane-line fitting result.

\section{Experimental Results and Analysis}

In this section, a series of experiments are presented to verify the proposed algorithm. The goal is to improve the robustness and efficiency of lane line detection in complex environments and visualize lane line areas. The video 
frames set for the experiment are collected from the data recorded by the driving recorder in a car, with the size of $768 \times 432$ a frame. All the simulations are run on Intel (R) Core (TM) CPU I5 8500 @ 3.00Ghz with a 16 GB RAM.

\subsection{Lane Line Detection Result}

When collecting experimental data, all the data contains lane line information, though there is a variety of interference. 12410 image frames are collected to evaluate the robustness of the proposed algorithm. The sections involve a wide variety of road conditions such as yellow lane, strong light, tree shadow, curve lane line and road traffic sign. Then, random gaussian noise is added to one of type. The lane line detection results are shown in Fig. 9(a)-(f). (a) is normal road, (b) add noise on the basis of (a), (c) exist yellow lane line, bright light in (d), trees cast shadows on the road in (e), there are traffic signs on the road in (f). As can be observed from the (b), despite there exist Gaussian noise, the proposed method can successfully identify the lane lines. (c) is the result of yellow lane condition. The impact of uneven light condition is reduced in (d). When facing complex roadside shadow in (e), the proposed algorithm performed well. The result of (f) demonstrated that the proposed algorithm reduced the impact of road traffic sign interference.

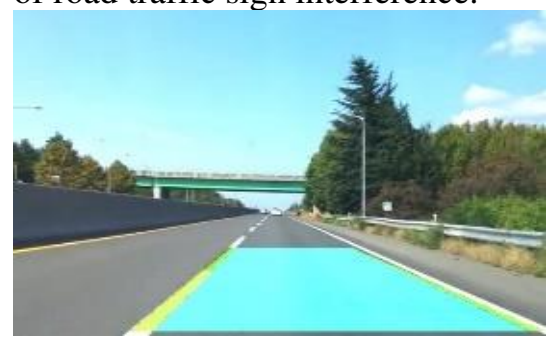

(a)

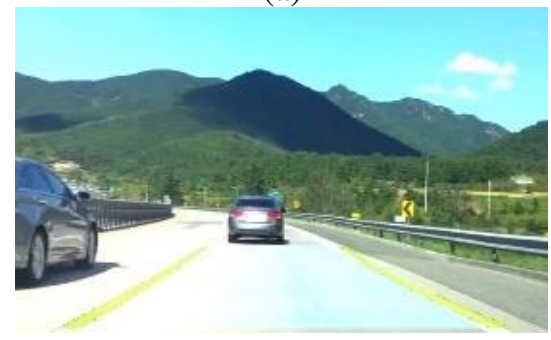

(d)

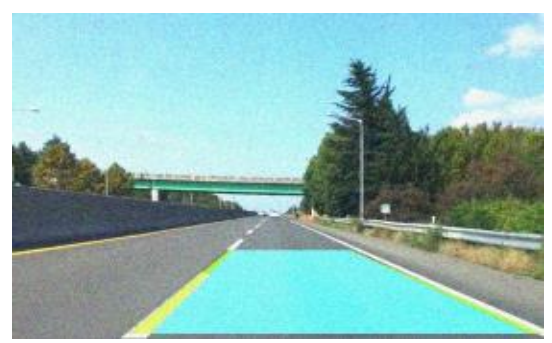

(b)

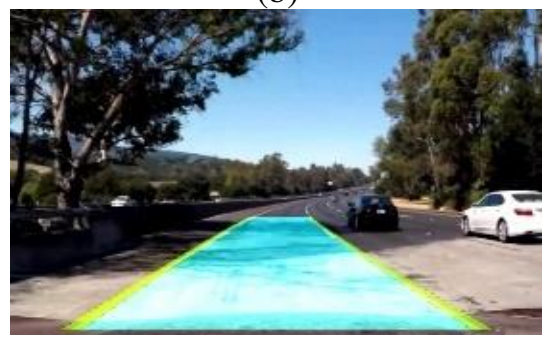

(e)

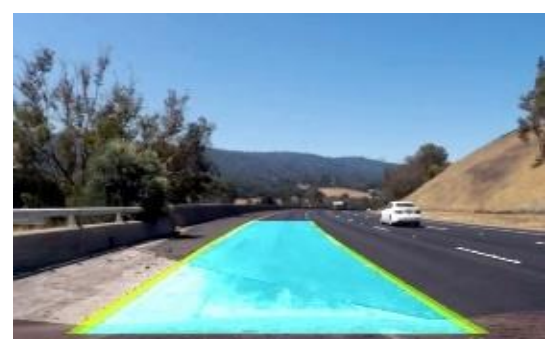

(c)

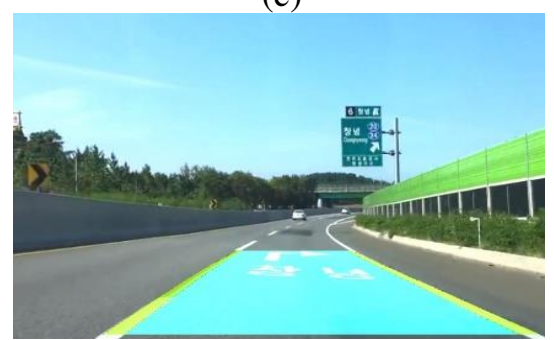

(f)

Figure 9 Detection samples show robustness in presence of gaussian noise, yellow lane, bright light, roadside shadow and road traffic sign ((a) Normal road, (b) Gaussian noise, (c) Yellow lane, (d) Bright light, (e) Roadside shadow, (f) Road traffic sig).

During the test, the accuracy of the algorithm is evaluated by comparing the difference between the detection result and the ground truth. The correct rate of the lane line detection is the ratio of the correctly detected lane number of frames and the total number of frames. The experimental results are shown in Table 1 and Figure 9. When the car is driving, the algorithm is less affected by noise, marking interference, yellow lane lines, shadow interference and bright light. It has certain accuracy and robustness.

Table 1 The detection accuracy in different driving environments

\begin{tabular}{llll}
\hline Road type & Total & False rate $(\%)$ & Correct rate $(\%)$ \\
\hline Normal & 8210 & 6.32 & 96.68 \\
Gaussian noise & 8210 & 8.66 & 91.34 \\
Yellow lane & 1800 & 5.00 & 95.00 \\
Bright light & 800 & 7.40 & 92.06 \\
Roadside shadow & 800 & 7.26 & 93.74 \\
Road traffic sign & 800 & 5.79 & 94.21 \\
\hline
\end{tabular}

\subsection{Comparison with other Lane Line Detection Method}

The next experiments are to demonstrate the prominent robustness and instantaneity of LFPE by comparing with Canny [16], Otsu [17], Optical Flow [18] and LFSW. The test data uses the same sample (4230 frames of effective video on a normal road). The LFSW and LFPF is more accurate, because the binary image of the lane line obtained by Multi-Feature fusion algorithm is clear, and pixel points found by window searching are more accurate. The Canny algorithm is more time-consuming because of the repetitive adoption of the Hough transform for line calculation. Therefore, LFSW and LFPE outperforms all the other alternative methods. 
Through statistical comparison of the experimental results in Table 2, the LFSW are superior to the Canny, Optical Flow and Otsu, and has highest accuracy with $96.68 \%$. Since the multi-feature fusion algorithm is used to obtain a high-quality binary image of lane-lines, and the feature extraction window is performed a refined lane-line pixel search. Moreover, the results from Table 2 show that the accuracy of the LFPF is $2.33 \%$ lower than that of the LFSW, but the speed is improved by $14.2 \mathrm{~ms}$. Because the lane-line pixel search is limited in the neighborhood of the lane-lines detected before.

Table 2 Performance comparison between proposed method and other methods

\begin{tabular}{lll}
\hline Method & Average accuracy $(\%)$ & Time $(\mathrm{ms})$ \\
\hline Canny [16] & 82.11 & 70.4 \\
Otsu [17] & 84.52 & 46.2 \\
Optical Flow [18] & 87.92 & 63.0 \\
LFSW & 96.68 & 34.8 \\
LFPF & 94.35 & 20.7 \\
\hline
\end{tabular}

\section{Conclusion}

In this study, a novel lane line detection based on multi-feature fusion and windows searching is proposed. At first, the multi-feature fusion method is used to separate the lane line from the complex background, then the pixel density distribution map and window search are used to determine the position of the lane line pixel, and finally the quadratic polynomial is selected to fit the lane line model, and the least square method is used to obtain parameters to improve the real-time performance of the algorithm. The proposed algorithm overcomes the shortcomings of conventional lane algorithms in the camera plane such as the difficulties of representing the lane model intuitively and the lack of wider-range lane data from a single image. It also improves anti-interference in lane detection. Finally, the algorithm also builds a lane prediction model for tracking lane detection. Several road scenes are selected for lane detection and the results indicate the effectiveness and robustness of the algorithm proposed in this paper. In addition, the algorithm is compared with three typical algorithms - The Canny, Otsu, and Optical flow algorithms - in terms of lane detection results. Several sections of freeways selected for testing, and statistics show that the algorithm proposed in this paper is better than the three conventional algorithms in terms of detection rate and reliability, the accuracy of the proposed algorithm is $96.68 \%$. At the same time, it is less time-consuming, has good timeliness, the average time for the proposed method to process a single frame of image is $20.7 \mathrm{~ms}$, and satisfies the motion planning for unmanned vehicle systems.

Although the algorithm proposed in this paper can achieve better results, some problems remain to be addressed. For instance, when a road surface takes an undulating form and fusion declines. In certain particular sections such as long-distance tunnels and others, the lane detection and tracking are weakened. In addition, the proposed method has a problem in detecting lane in sharp curves and in the presence of adjacent vehicles and pedestrians. In future research, multiple road features will be further combined with more sensor information to detect the sharply curved lanes amidst the presence of vehicles and enhance the flexibility and robustness of the proposed algorithm.

\section{Declarations}

\section{Availability of data and materials}

We can provide the data.

\section{Competing interests}

The authors declare that they have no competing interests.

\section{Funding}

This work is sponsored by the National Key R \& D Program of China (No. 2018YFB1004904), the Huai' an science and technology project (No. HAC201705, No.HAB201803), the key project of Jiangsu Provincial Department of Education (No.18KJA520001), Postgraduate Research \& Practice Innovation Program of Jiangsu Province (SJCX18_0901), six talent peaks project in Jiangsu Province (XYDXXJS-011), Jiangsu 333 engineering research funding project (BRA2016454).

\section{Authors' contributions}

CC conceived and designed the algorithm implementation. SG, ZP, HZ, ZH participated in the experiment and data processing and edited the manuscript. The final draft read and approved by all authors. 


\section{Acknowledgements}

The authors thank the editor and anonymous reviewers for their helpful comments and valuable suggestions.

\section{Authors information}

Chuangxin Cai received the BS degree in mathematics from the Nanjing Xiaozhuang University in 2018. He is currently a graduate Student in the Master of the Computer Science Department with Huaiyin Institute of Technology. Email: 1157981131@qq.com

Shangbing Gao received the BS degree in mathematics from the Northwestern Polytechnical University in 2003. He received the MS degree in applied mathematics from the Nanjing University of Information and Science and Technology in 2006. He received the Ph.D. degree with School of Computer Science and Technology, Nanjing University of Science and Technology (NUST). He is currently a Professor of the Computer Science Department with Huaiyin Institute of Technology. He has authored over 50 scientific papers. Some of them are published in the IEEE TRANSACTIONS ON NEURAL NETWORKS AND LEARNING SYSTEMS, and the IEEE TRANSACTIONS ON CIRCUITS AND SYSTEMS FOR VIDEO TECHNOLOGY. His current research interests include machine learning, data mining, and pattern recognition. Email: luxiaofen_2002@126.com

Zhigeng Pan He got his Ph.d degree in 1993, and he became a full professor in Zhejiang University in 1996 because of his excellent academic performance. He has published more than 100 technical papers on important journals (such as PAMI, TVCG, IEEE Multimedia) and conferences (such as ACM Multimedia, IEEE VR, et al ). He is a member of IEEE, ACM SIGGRAPH. His research interests include virtual reality, computer graphics and HCI. Currently, he is the Editor-in-Chief of $\langle\langle$ Trnsactions on Edutainment $\rangle\rangle$. Now he is Director, Digital Media and HCI Research Center, Hangzhou Normal University.Email:zgpan@hznu.edu.cn

Hao Zheng is a postdoc in the Department of Computer Science and Engineering at Southeast University, Nanjing, China. His research interests include pattern recognition, image processing, face recognition, and computer vision. Zheng received a $\mathrm{PhD}$ in pattern recognition and intelligence systems from the Nanjing University of Science and Technology. Contact him at 30741989@qq.com.

Zihe Huang received the BS degree in mathematics from the Huaiyin Institute of Technology in 2017. He is currently a graduate Student in the Master of the Computer Science Department with Huaiyin Institute of Technology. Email: jsshuangzihe@163.com

\section{References}

[1] Piao C, Lu C. Lane Detection of Unstructured Roads Based on WS-P2PNet[C]// 2019 International Conference on Intelligent Computing, Automation and Systems (ICICAS), 2019:596-599.

[2] Perng J W, Hsu Y W, Yang Y Z, et al. Development of an embedded road boundary detection system based on deep learning[J]. Image and Vision Computing, 2020, 100:103935.

[3] Zang J, Zhou W, Zhang G, et al. Traffic Lane Detection using Fully Convolutional Neural Network[C]// 2018 Asia-Pacific Signal and Information Processing Association Annual Summit and Conference (APSIPA ASC),2018:305-311.

[4] Deng G, Wu Y. Double Lane Line Edge Detection Method Based on Constraint Conditions Hough Transform[C]// 2018 17th International Symposium on Distributed Computing and Applications for Business Engineering and Science (DCABES), 2018:107-110.

[5] Zhang X, Zhu X. Autonomous Path Tracking Control of Intelligent Electric Vehicles based on Lane Detection and Optimal Preview Method[J]. Expert Systems with Applications, 2018, 121:38-48.

[6] Zarbakht N, Zou J. Lane Detection Under Adverse Conditions Based on Dual Color Space[C]// 2018 Digital Image Computing: Techniques and Applications (DICTA), 2018:1-5.

[7] Baili J, Marzougui M, Sboui A, et al. Lane departure detection using image processing techniques[C]// 2017 2nd International Conference on Anti- 
Cyber Crimes (ICACC), 2017:238-241.

[8] Jide Q, Bing C, Jiye Q. Fast Lane Detection Algorithm Based on Region of Interest Model[J]. Journal of University of Electronic Science and Technology of China, 2018, 47(03):356-361.

[9] Ajmal A, Hollitt C, Frean M, et al. A Comparison of RGB and HSV Colour Spaces for Visual Attention Models[C]// 2018 International Conference on Image and Vision Computing New Zealand (IVCNZ), 2018:1-6.

[10] Singh T. Foggy Image Enhancement and Object Identification by Extended Maxima Algorithm[C]// 2017 International Conference on Innovations in Control, Communication and Information Systems (ICICCI), 2017:1-5.

[11] Singh K B, Mahendra T V, Kurmvanshi R S, et al. Image enhancement with the application of local and global enhancement methods for dark images[C]// 2017 International Conference on Innovations in Electronics, Signal Processing and Communication (IESC), 2017:199-202.

[12] Quach C H, Tran V L, Nguyen D H, et al. Real-time lane marker detection using template matching with RGB-D camera[C]// 2018 2nd International Conference on Recent Advances in Signal Processing, Telecommunications \& Computing (SigTelCom), 2018:152-157.

[13] Muthalagu R, Bolimera A, Kalaichelvi V. Lane detection technique based on perspective transformation and histogram analysis for self-driving cars[J]. Computers \& Electrical Engineering, 2020, 85(07):106653-106663.

[14] Deng G, Wu Y. Double Lane Line Edge Detection Method Based on Constraint Conditions Hough Transform[C]// 2018 17th International Symposium on Distributed Computing and Applications for Business Engineering and Science (DCABES), 2018:107-110.

[15] Hajjouji I E, Mars S, Asrih Z, Mourabit A E. A novel FPGA implementation of Hough Transform for straight lane detection[J]. Engineering Science and Technology, 2019, 23(05):274-280.

[16] Jiang L, Li J, Ai W. Lane Line Detection Optimization Algorithm based on Improved Hough Transform and R-least Squares with Dual Removal[C]// 2019 IEEE 4th Advanced Information Technology, Electronic and Automation Control Conference (IAEAC), 2019:186-190.

[17] Ma L Y, Hua C S, He Y Q, et al. A Lane Detection Technique Based on Adaptive Threshold Segmentation of Lane Gradient Image[C]// 2018 4th Annual International Conference on Network and Information Systems for Computers (ICNISC), 2018:182-186.

[18] Zhuang B, Duan J, Zheng B, et al. Algorithm Research of Fast Lane Detection Based on Optical Flow[J]. Computer Measurement \& Control , 2019, 27(09):146-150. 


\section{Figures}

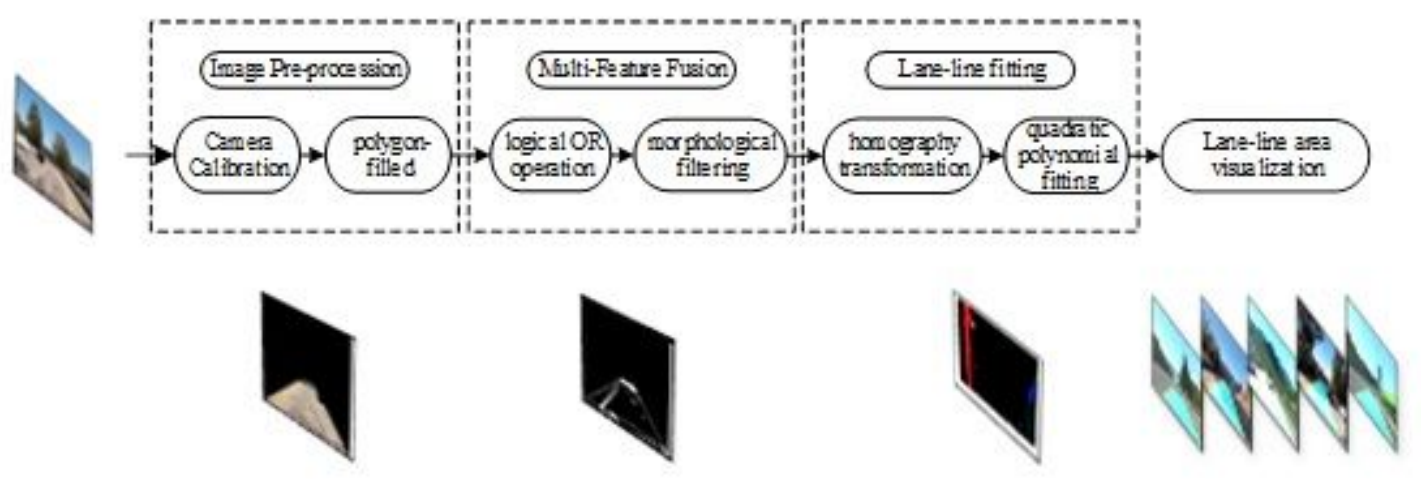

\section{Figure 1}

Flowchart of proposed lane detection method.

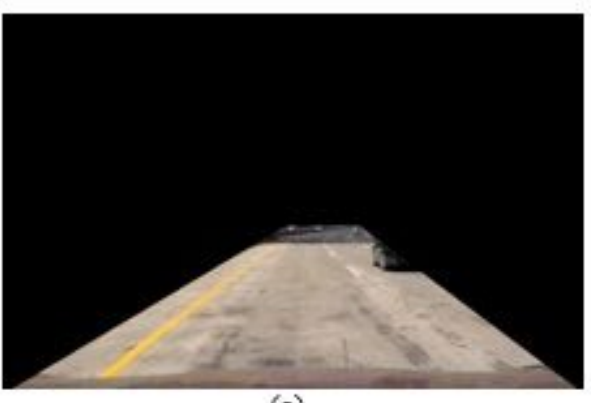

(a)

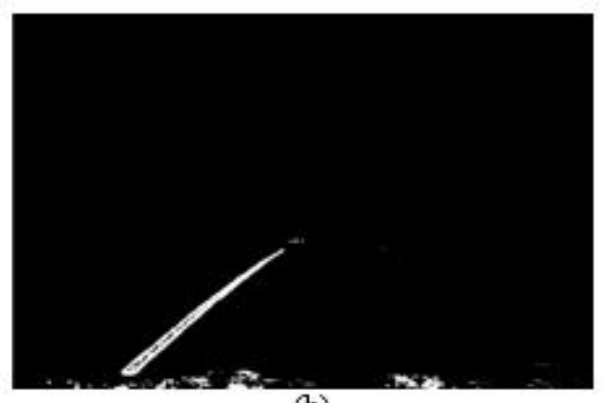

(b)

Figure 2

Yellow lane extraction ((a) Input image with region of interest, (b) Yellow mask).

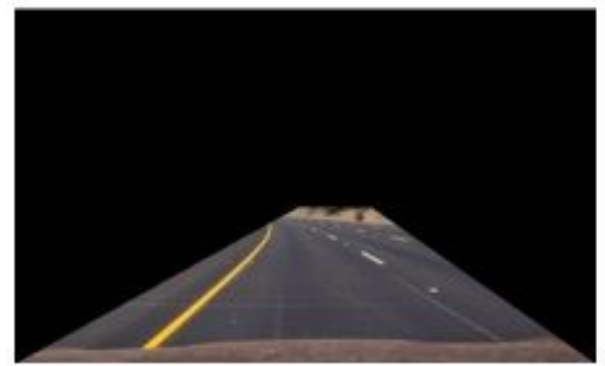

(a)

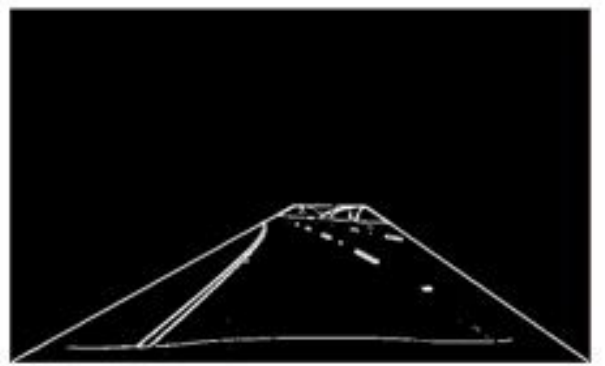

(b)

\section{Figure 3}

Image filtered with gradient operator ((a) Input image with region of interest, (b) Sobel operator). 


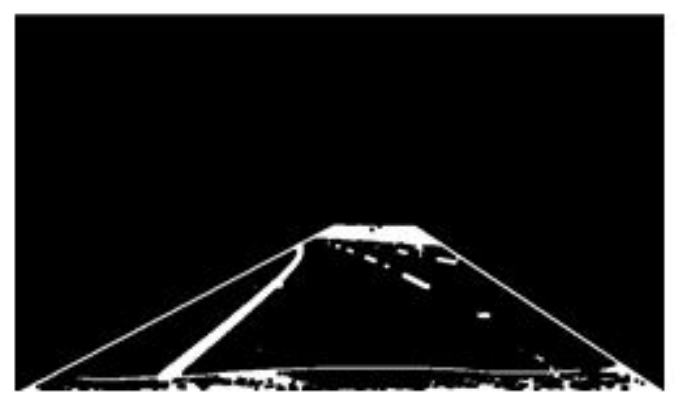

\section{Figure 4}

The result of the multi-feature fusion algorithm under road condition.

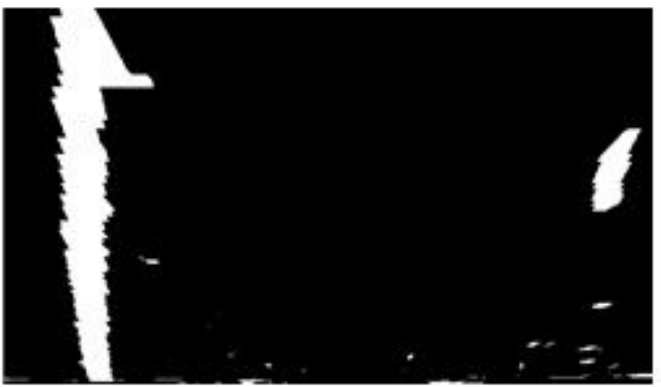

Figure 5

The Image of homography transformation.

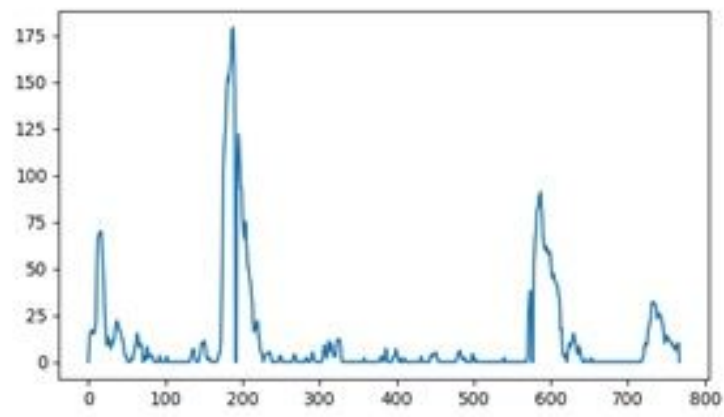

\section{Figure 6}

The density distribution of pixel density. 


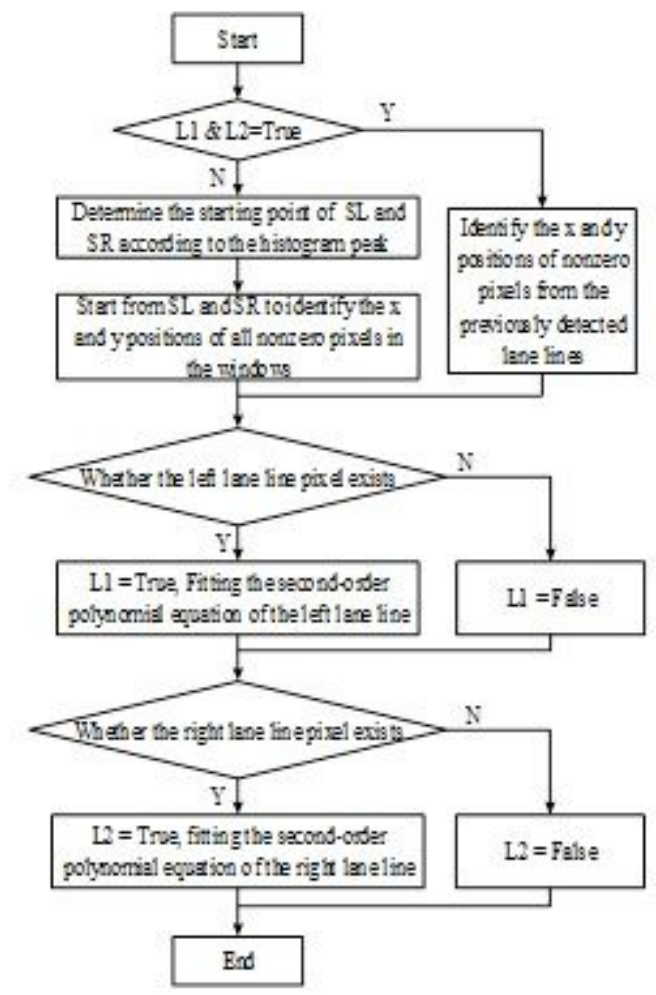

Figure 7

The flow chat of lane-line pixel search algorithm.

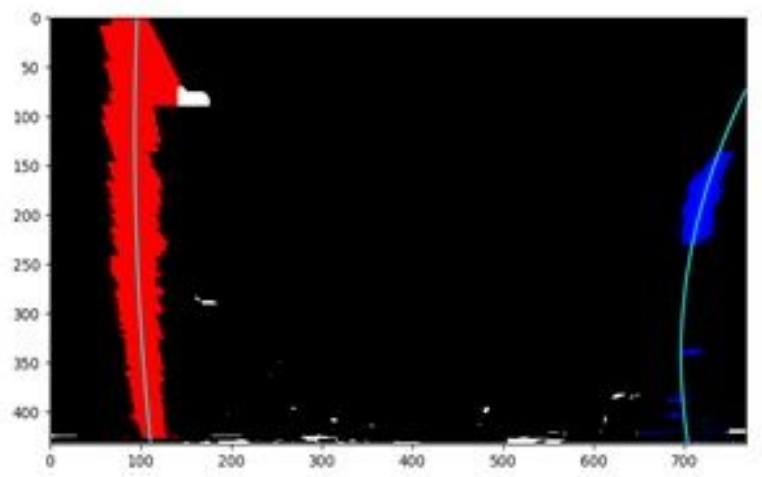

\section{Figure 8}

The lane-line fitting result. 


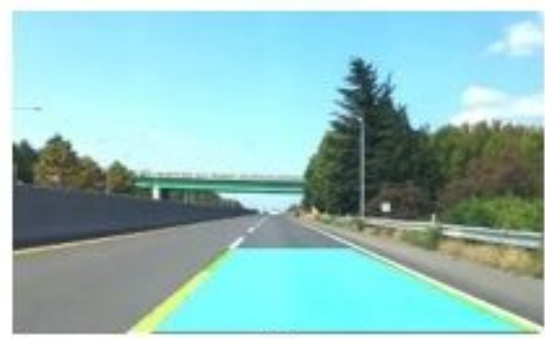

(a)

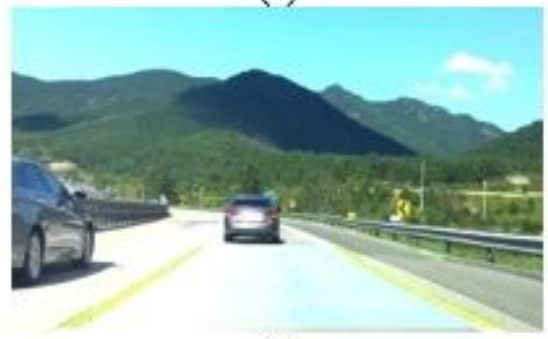

(d)

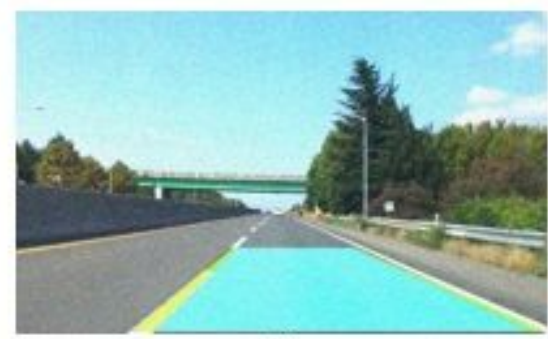

(b)

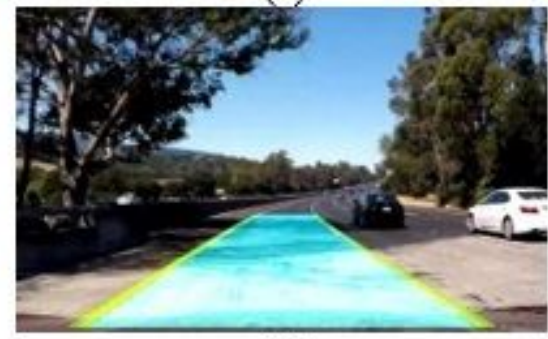

(e)

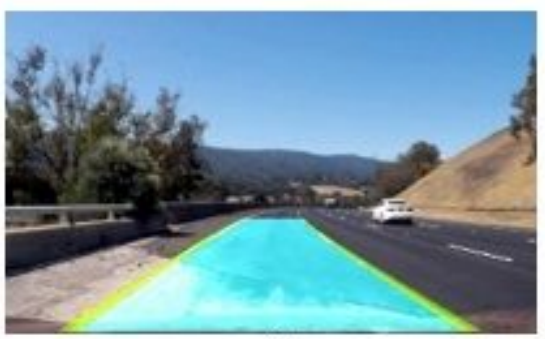

(c)

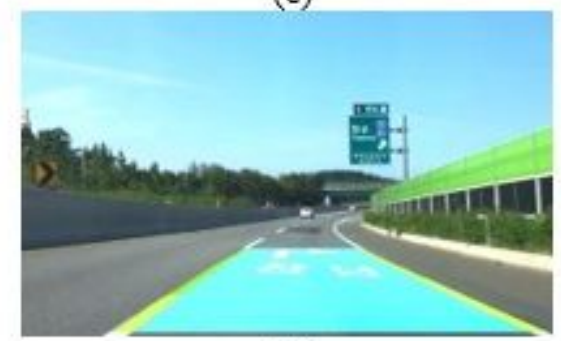

(f)

Figure 9

Detection samples show robustness in presence of gaussian noise, yellow lane, bright light, roadside shadow and road traffic sign ((a) Normal road, (b) Gaussian noise, (c) Yellow lane, (d) Bright light, (e) Roadside shadow, (f) Road traffic sig). 